DANIELLA PARODI MIRANDA

SANTIAGO, CHILE

DANIELLAGPM@GMAIL.COM

\section{Material compuesto a partir del residuo cáscara de nuez Juglans regia}

\author{
Composite material from Juglans regia shell residue
}

Resumen. El trabajo se realizó con el objetivo de desarrollar un innovador material compuesto que presenta como elemento principal la cáscara de nuez de nogal, un residuo de la agroindustria chilena. Se centra en una investigación experimental que constó de tres fases: en la primera se estudió la cáscara y se trituró para conseguir diversas granulometrías, en la segunda se mezcló con diversos aglutinantes biodegradables en distintas proporciones. Después de combinar estas variables se elaboraron probetas de estudio para luego en la tercera fase caracterizar el material a través de diversos ensayos y pruebas. Finalmente, se analizó el ciclo de vida del proceso del material con el fin identificar posibles ineficiencias en su etapa de diseño.

Los resultados muestran que es un material liviano con destacadas características de aislación térmica, que se puede conformar a partir de moldes y herramientas comunes en la industria. Además de lo anterior, requiere bajo nivel de tecnología y energía para ser procesado, genera una mínima cantidad de desperdicios y posee un ciclo de vida circular, por lo que podría presentar una gran oportunidad para el desarrollo de nuevos productos. Palabras clave: cáscara de nuez, diseño sostenible, experimentación de materiales, material compuesto.

\begin{abstract}
The work was done with the aim of developing an innovative composite material whose main element would be the shell of the walnut nuts, a Chilean agro-industry remnant. The experimental investigation, on which the work was focused, consisted of three phases: on the first one the nutshell was studied and triturated in order to obtain different granulometries. On the second one, it was mixed with different biodegradable binder agents in several proportions. After combining these variables, test tubes were prepared to characterize the material through different trials and tests on the third phase. Finally, the material process life cycle was analyzed with the purpose of identifying eventual inefficiencies during the design stage. The results show that it is a light material, with outstanding thermal insulation characteristics, that can be produced with common tools and molds in the industry. Moreover, the manufacturing process does not require a high level of technology and energy, it generates a minimal quantity of waste, and since it possesses a circular life cycle, it might allow the opportunity to the development of new products.
\end{abstract}

Keywords: composite material, experimentation of materials, nutshell, sustainable design 


\section{Introducción}

Si por unos minutos nos detenemos a mirar nuestro planeta, nos daremos cuenta de que existe un gran deterioro medio ambiental y un desequilibrio en la interacción de los seres humanos entre ellos y con su entorno. Por lo tanto, aspirar a una cultura de desarrollo sostenible es un reto para todos quienes vivimos en el planeta Tierra, reto que no había sido tratado como un modelo de desarrollo universalmente aceptado hasta que la comisión Brundtland en 1987 publicara el informe "Our Common Future". En el informe se define desarrollo sostenible como aquel que satisface las necesidades del presente sin comprometer la capacidad de las generaciones futuras de satisfacer sus propias necesidades (Brundtland, 1987; Capuz \& Gómez, 2004). Actualmente, distintas instancias, normativas y cambios en la manera de pensar han llevado a que el hombre comience a ser consciente de sus actos y las consecuencias con el entorno, pues el desarrollo social y económico de los seres humanos depende de la mejor utilización de los recursos disponibles (Ndazi, Tesha, \& Bisanda, 2006). Estos cambios han generado nuevas tendencias al momento de consumir, dando importancia a los productos que consideran aminorar el impacto al medio ambiente. Según la Agencia Federal del Medio Ambiente, gran parte de la carga ambiental de un producto o servicio se define al diseñar un producto, por tanto, el problema de generación de residuos, el impacto de estos en el medio ambiente y la salud humana es consecuencia de un proceso que tiene como etapa inicial su diseño. Por este motivo, se entiende que la implicancia del diseñador va más allá de la creación de productos o servicios, pues puede contribuir en aspectos fundamentales de la vida humana ya sea en lo social, ambiental o cultural. Los diseñadores pueden mejorar la calidad de vida e influir en el uso sostenible de los recursos a través de sus decisiones (Karana, Pedgley, \& Rognoli, 2014). Como respuesta a la necesidad de cambio que existe, comienza a darse más cabida al diseño sostenible y al diseñador como agente para un desarrollo sostenible. Por esta razón, esta investigación se encuentra dirigida a dar uso a los recursos que hoy son considerados como residuos y desarrollar un material que posee un ciclo circular, de la cuna a la cuna, ya que al finalizar su vida útil pasan a ser nutrientes de otros ciclos sin llegar a transformarse en residuo, sino que en un recurso (McDonough \& Braungart, 2005) para su aplicación en productos. Se debe tener en cuenta que se consideran materiales y productos sostenibles a aquellos que minimizan el uso de recursos, tienen impactos ecológicos bajos y no representan riesgos o muy bajos riesgos para la salud humana o el medio ambiente (Calkins, 2009). Una de las funciones del Diseño es abrir puertas, jugar con lo inusual y lo no probado, además las competencias en el diseño de productos confieren la capacidad de transformar los recursos naturales en productos negociables; recursos que tienen un potencial en la reducción de costes y peso (Karana et al., 2014), pero que también generan menor impacto ambiental. Por otra parte, se ha detectado que la industria chilena de frutos secos incluye como principales especies las nueces de nogal y almendras. Las nueces forman parte de un mercado que mundialmente ha experimentado un aumento sostenido en los últimos años. Su consumo se ha incrementado desde fines de los años 90 como resultado del aumento del ingreso y la tendencia al consumo de productos naturales, sobre todo en los países más ricos (Fundación para la Innovación Agraria, 2007). Esta situación ha repercutido de buena manera en Chile, convirtiéndolo en el séptimo productor mundial de nueces con cáscara, con una producción anual al año 2014 de 58.909 Toneladas (FAO, 2017), lo que deja como 
residuo alrededor de 22.784 toneladas de cáscara al año (iQonsulting, 2016). De esas toneladas de residuos, un $15,5 \%$ se va a vertederos (IdeaConsultora, 2012), lo cual significa un costo para la industria. Sin embargo, lo más común es que la cáscara se transforme en un descarte durante toda la temporada de partido, mayormente en la época de cosecha y poscosecha, lo cual es un problema de higiene, ya que se acumula en patios atrayendo roedores y polillas y al captar humedad se generan hongos (Guzmán, 2016). Se ha detectado que actualmente no se le ha dado la importancia adecuada como materia prima. Se utiliza principalmente para rellenar caminos, se tira a la tierra para devolver sus nutrientes como abono o también para formar una cubierta protectora manteniendo la humedad al disminuir la evaporación, además se utiliza como abrasivo, en forma de polvo, en las industrias. La cáscara de nuez seca es conocida entre los productos de biomasa por su potentísimo poder calórico, pudiéndose utilizar como pellet (Marlenin, 2011; Torres, 2010). Existen también empresas tales como Econut y Pacific Nut que venden la cáscara de nuez para usos en biocombustible, el precio de compra fluctúa entre CLP \$10 y CLP \$13 por kilo. Sin embargo, al darle un uso más elaborado a este residuo se podría generar un incremento en el precio de venta, lo que otorga una oportunidad de nuevos negocios para la empresa productora de nuez, al sacar el residuo de un proceso lineal para reintegrarlo como nutriente en un nuevo proceso productivo. Se toma en consideración, por una parte, que en las últimas décadas han surgido centros de conocimiento que impulsan la integración de los materiales y los diseñadores para crear nuevos materiales y procesos de manufactura innovadores (Ramirez \& Wechsler, 2010; Valencia, 2008), y por otra, que ya existen investigaciones previas de desarrollo de materiales compuestos basados en cáscara de nuez. En Turquía han estudiado la factibilidad de manufacturar tableros a través de la experimentación con tres adhesivos y dos densidades diferentes, concluyendo que sus características seguían en promedio con otros tableros de partículas manufacturados con subproductos de la agricultura (Çöpür, Güler, Akgül, \& Taş̧̧ığlu, 2007). También se ha estudiado el potencial uso de las cáscaras de nuez como materia prima para ser utilizada en una mezcla con partículas de madera en la fabricación de paneles, para conocer la factibilidad de uso de las partículas de cáscaras de nuez en la manufactura de tableros de tres capas. Los resultados han mostrado que es posible producir tableros al mezclar partículas de cáscaras de nuez y madera, utilizando como adhesivo urea formaldehído (Pirayesh, Khazaeian, \& Tabarsa, 2012), que al añadir partículas de cáscara de nuez mejora la resistencia al agua de los paneles, sin embargo, las propiedades mecánicas disminuyen con el incremento de partículas de cáscaras en el contenido de los tableros. Por otra parte, Nitin and Shingh mezclaron polvo de cáscara de nuez con resina epoxi en la producción de paneles y concluyeron que la densidad obtenida es muy adecuada para aplicaciones ligeras y que la resistencia a la tracción es suficiente para materiales que reemplazan la madera (Nitin \& Singh, 2013). Es aquí donde se ha encontrado una importante oportunidad, por tanto se propone el aprovechamiento del residuo de cáscara de nuez desde la mirada innovadora del diseñador, para ser utilizado como materia prima principal en el desarrollo de un nuevo material compuesto con un adhesivo biodegradable, desarrollo que considera el ciclo de vida del material y el cómo se utilizará, en base a lo cual se pretende que pueda ser trabajable con herramientas que se utilizan comúnmente, moldeable, que no genere residuos y permita proponer aplicaciones de diseño. 
Tabla 1. Composición cáscara de nuez ( $\mathrm{g} / 100 \mathrm{~g}$ de peso seco)

\section{Cáscara de nuez de nogal Juglans regia.}

El nogal es un frutal que ha acompañado al hombre a través de toda su historia, es un frutal antiguo, en Europa se han encontrado restos que datan de la edad del hierro, pertenece al género Juglans, nombre entregado por los romanos (Lemus, 2001).

La cáscara de nuez corresponde a un residuo sólido agrícola que deriva de la etapa de partido en el procesamiento de la nuez, representa entre el $50 \%$ del peso total de la nuez seca (iQonsulting, 2016).

Según el estudio "Citric acid modified agricultural by-products as copper ion adsorbents" se estima que la cáscara de nuez posee como componentes estructurales celulosa, hemicelulosa y lignina, como se muestra en la Tabla 1 , por tanto, se trata de un material lignocelulósico según el estándar (Wartelle \& Marshall, 2000). La celulosa y hemicelulosa corresponden a polímeros naturales y la lignina, la segunda materia biológica más abundante en el mundo, a un heteropolímero, considerada como una de las moléculas responsables de otorgar mayor dureza a las maderas (Trujillo \& Arias, 2013).

\section{\begin{tabular}{|l|l|l|l|l} 
Celulosa (\%) & Hemicelulosa (\%) & Lignina (\%) & Proteína (\%) & Ceniza (\%)
\end{tabular}}

\begin{tabular}{l|l|l|l|l}
60.2 & 13.2 & 18.6 & 1.3 & 1.1
\end{tabular}

Otras características que se han estudiado indican que su densidad aparente corresponde a $650 \mathrm{~kg} / \mathrm{m}^{3}$, su dureza es de 2,5 Mohs, posee un pH neutro, su peso específico es $1,25 \mathrm{~kg} / \mathrm{l}$ y su autoignición ocurre por encima de los $270^{\circ} \mathrm{C}$ (Torres, 2010; Wartelle \& Marshall, 2000). En cuanto a su descomposición, es biodegradable y al mantener la cáscara de nuez con humedad se torna de color verde a negro en un lapso no mayor a 6 meses, y así se puede realizar compostaje (Romero et al., 2012).

La cáscara requiere bajo nivel de tecnología y energía para ser reprocesado, pues se obtiene limpia y seca después de ser procesada. Además, cabe destacar que posee un bajo costo y posee disponibilidad durante todo el año. etapa del ciclo de vida

Materia Prima
Producción
Gestión de fin de vida
Tabla 2. Requerimientos para el compuesto
biodegradable de cáscara de nuez
Fuente: Parodi Miranda, 2016, basado en Calkins,
2009; McDonough \& Braungart, 2005

requerimiento

Material Local

Bajo costo

Materiales limpios

No tóxico

energía para procesamiento

gua para procesamiento

Bajo costo

No tóxico

Biodegradabilidad

\section{descripción}

El material debe ser local o estar disponible en el lugar de producción

Dar preferencia a aquellos que tienen menor costo

Materiales poco contaminantes y que generen mínima cantidad de residuos Utilizar en mayor porcentaje el residuo agroindustrial Inocuo a la salud humana

Bajo nivel de energía o uso de energías renovables para su procesamiento

Procesos que minimicen o prevengan el uso de agua

Dar preferencia a aquellos que tienen menor costo

Inocuo a la salud humana

Debe gestionarse su fin de vida a través de la biodegradabilidad

\section{Desarrollo del material}

Los requerimientos para el desarrollo del material se presentan en la Tabla 2, para definirlos se toman como base los tres pilares de la sostenibilidad, los cuales corresponden al económico, social y medioambiental, además se consideran las estrategias del ecodiseño y el ciclo de vida del material, de tal manera que se pueda conseguir un ciclo circular de la cuna a la cuna. 


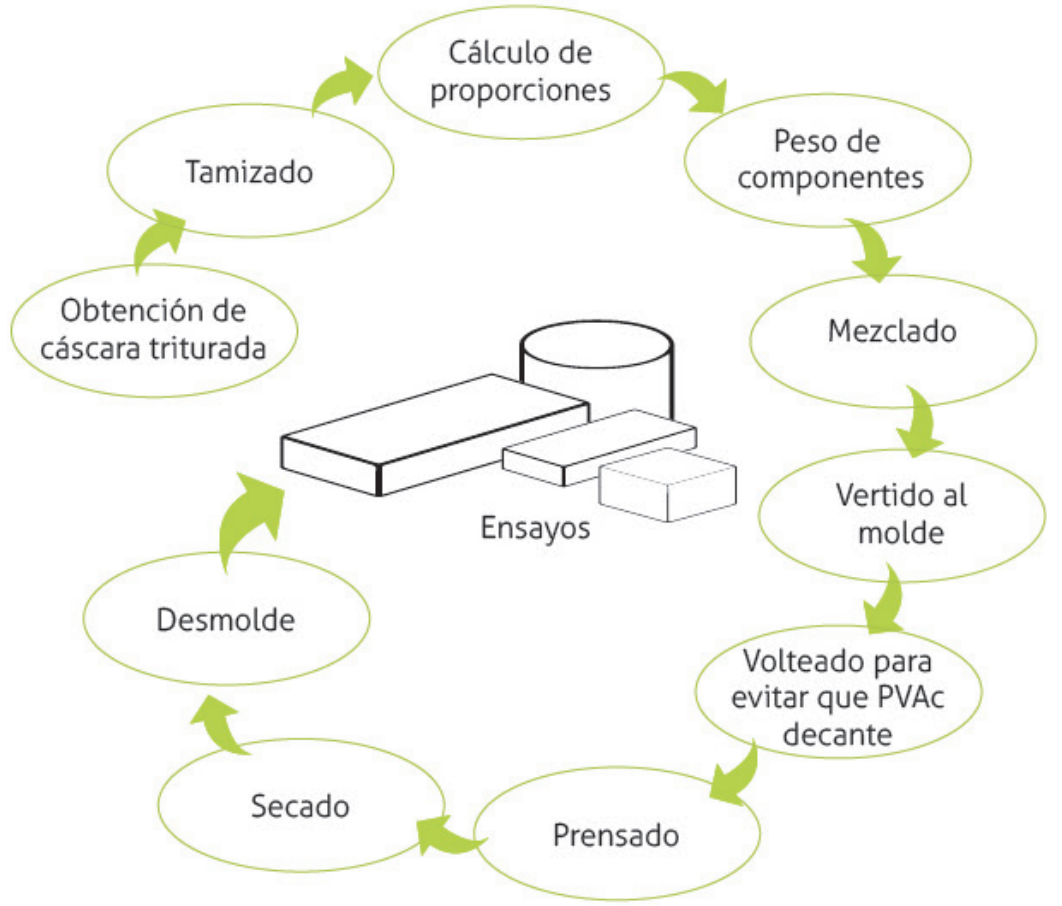

\section{Fases de la experimentación}

La investigación consistió en desarrollar un estudio práctico mediante la experimentación con el residuo cáscara de nuez y diversos aglutinantes biodegradables con el fin de desarrollar un material compuesto a partir de la utilización del residuo cáscara de nuez como materia principal.

En la primera fase se buscó cumplir con el objetivo de establecer el proceso de elaboración de un material compuesto como se muestra en Figura 1, el cual se desarrolló en base al residuo cáscara de nuez y un aglomerante biodegradable. Para dar cumplimiento, se realizó una etapa de experimentación, la cual consistió en triturar la cáscara con un molinillo eléctrico para maíz. Una vez triturada la cáscara se tamizó para conseguir una granulometría de 1 a $2 \mathrm{~mm}$, que luego se mezcló manualmente con PVAC como aglomerante y se determinó como mejor proporción la de 60-40\%, la cual era la mayor parte de la cáscara.

\section{Ensayos}

En la fase dos, con el objetivo de evaluar las características físico-mecánicas del material, el compuesto se somete a diversos ensayos.

Respecto a los ensayos físicos, se realizaron de densidad, absorción de agua e hinchamiento, los cuales fueron determinados de acuerdo a la norma ASTM D1037-99, norma de método de prueba para evaluar propiedades de materiales basados en fibra de madera y tableros de partículas (ASTM, 1999). Se ensayaron cinco probetas de $50 \times 50 \times 3 \mathrm{~mm}$, para calcular la densidad, las probetas fueron secadas por 24 horas a $103^{\circ} \mathrm{C}$ en estufa marca Blinder. Por su parte, para el ensayo de absorción e hinchamiento las probetas se sumergieron horizontalmente en agua, y se evaluaron transcurridas 2 horas y luego de 24 horas.
Figura 1. Diagrama del proceso de elaboración del material. Fuente: Parodi Miranda, 2016. 
Para los ensayos mecánicos, se utilizó una máquina Instron de la Universidad Tecnológica Metropolitana (UTEM), la cual tiene una velocidad de trabajo de $2 \mathrm{~mm} / \mathrm{min}$. Se realizaron ensayos de flexión, compresión y tracción. Los tamaños de las probetas corresponden a $22 \times 140 \times 10 \mathrm{~mm}, 20 \times 100 \times 5 \mathrm{~mm}$ y $20 \times 100 \times 5 \mathrm{~mm}$, respectivamente, y se evaluaron mínimo 5 probetas en cada ensayo. Para el ensayo de flexión, la luz (distancia entre puntos de apoyo) fue de $10 \mathrm{~mm}$, para compresión y tracción, las mordazas se ubicaron a $10 \mathrm{~mm}$. Posteriormente, el material se sometió a pruebas de mecanizado y se tomó como referencia la norma ASTM D1666-11, norma de método de prueba para la realización de pruebas de mecanizado en madera y materiales de paneles basados en madera (ASTM, 2011). El equipamiento utilizado corresponde al que se encontraba disponible para los estudiantes en la Facultad de Arquitectura y Urbanismo de la Universidad de Chile. Se realizaron pruebas de corte, perforación y torneado, el equipamiento utilizado en cada uno de los ensayos de maquinado corresponde respectivamente a esmeril, sierra ingleteadora, sierra de huincha, corte láser, luego taladro manual, de pedestal y torno para madera. Se someten a ensayo cinco muestras de $150 \times 70 \mathrm{~mm}$ con espesor de $10 \mathrm{~mm}$. La evaluación se realiza a partir de una inspección visual. Por tanto, a partir de la observación del acabado de la superficie, se utiliza la clasificación del maquinado propuesto por Frühwald y otros en 1992 (Rodríguez et al., 2015), en la que existen cinco clases definidas según la magnitud de los defectos.

Para conocer cómo se comporta térmicamente el compuesto, se ensaya a partir del proyecto de tesis de estudiantes de Ingeniería de Ejecución en Climatización de la Universidad de Santiago (USACH), que tiene como objetivo diseñar un montaje experimental que permita el estudio del comportamiento térmico convectivo de un material constructivo. Para llevarlo a cabo se implementó un ducto de sección cuadrada de $10 \mathrm{~cm}$ y 2 metros de largo, en el cual se insertan las probetas: una en posición horizontal de $40 \times 40 \mathrm{~mm}$ paralela al flujo y la otra al final del ducto en posición vertical de $40 \times 50 \mathrm{~mm}$, la cual se ubica perpendicular al flujo. Una vez ubicadas, se agrega una carga térmica convectiva al flujo para identificar el comportamiento de las probetas. La conductividad en las probetas se evalúa a $20^{\circ} \mathrm{C}$ y en base a la diferencia de temperatura registrada en cada cara de las probetas; el área y espesor. Se aplica la fórmula de conductividad considerando que para esta temperatura se utilizan $0,06 \mathrm{~kW}$ de potencia de trabajo (Sellao \& Castillo, 2015).

\section{Exploración formal}

En la fase tres, se busca evaluar las posibilidades constructivas del material compuesto a través de moldes de doble y triple curvatura, por tanto, se conforman piezas primero a través de moldes de fácil acceso y posteriormente se generan moldes de MDF en router CNC para explorar diversas morfologías. La mezcla es prensada en los moldes de manera manual con prensas sargentos. El procedimiento consiste en verter la mezcla y prensar por 1 hora, luego de haber transcurrido se aplica una temperatura de 35 a $40^{\circ} \mathrm{C}$ por 2 horas con ampolleta infrarroja a $250 \mathrm{~mm}$ de distancia, para luego continuar con su tiempo de secado de manera natural durante 9 horas (Parodi Miranda, 2016). 
evaluación

del material

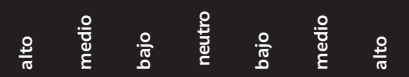

atributo/ requerimiento + atributo/ requerimiento -

Brillante
$\begin{gathered}\text { Cálido } \\ \text { Liviano }\end{gathered}$

Tabla 3. Forma del diferencial para análisis perceptual. Fuente: Aros, Narváez, \& Aros, 2009

\section{Análisis perceptual}

Al buscar una caracterización más integral del material y pensando en una futura definición de usos y usuarios, se propone realizar un análisis perceptual, en la cual se utiliza la herramienta de diferencial semántico que se presenta en la Tabla 3.

La muestra consiste en treinta personas que no tienen conocimientos previos sobre el material que se les presenta, de estas, quince personas poseen conocimientos previos sobre materiales, pues corresponden a diseñadores, arquitectos, artistas $u$ otros que se desempeñan en el ámbito de los materiales y la otra mitad son ajenos al ámbito de los materiales. Para la realización de la prueba se optó por presentar el material en distintos formatos, con igual espesor, las cuales son entregadas a cada persona para que puedan interactuar. Los atributos que correspondan serán evaluados en un rango de 3 a -3, donde 3 representa el atributo deseable y -3 su contrario, es decir, el atributo que se considera indeseable.

\section{Fin de vida}

Para determinar si es posible cerrar su ciclo al terminar la vida útil, se realiza un ensayo de manera casera, que consiste en mantener probetas al interior del hogar y por otra parte se introducen probetas en un cajón de vermicompostaje con lombrices californianas (Eisenia foetida) obtenidas de la empresa Humus de Chile y en un macetero con tierra húmeda a la intemperie. Esto se realizó por un período de cuatro meses y se evaluó a partir de la observación. 
Figura 2. Diagrama ciclo de vida del material compuesto de cáscara de nuez.

Fuente: Parodi Miranda, 2016

Figura 3. Resultado del conformado del material compuesto de cáscara de nuez.

Fuente: Parodi Miranda, 2016

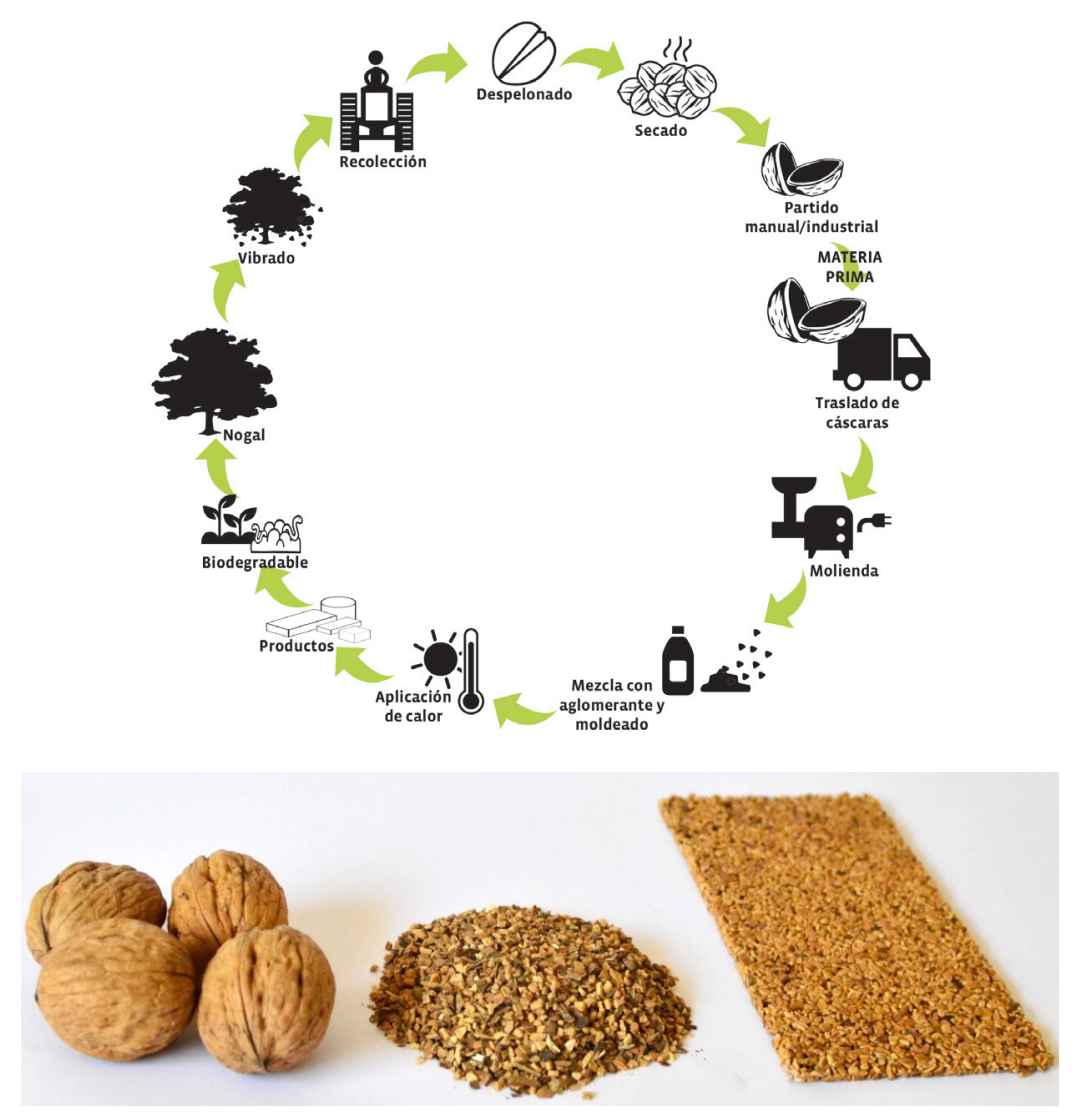

\section{Análisis de ciclo de vida}

En términos cualitativos, generalmente los impactos medioambientales de los tableros de partículas se basan en que provienen de fibras de madera de pinos de plantaciones de monocultivo (International, 2002; Magin, 2002) y por otra parte están dados por los adhesivos, que comúnmente corresponden a urea formaldehído, basado en derivados del petróleo y cancerígenos (EPA, 2016; Rivela, Hospido, Moreira, \& Feijoo, 2006). También hay que sumarle a esto la alta cantidad de energía requerida y emisiones de $\mathrm{CO}_{2}$ generadas durante el proceso de secado y preparación de las fibras de madera y en el proceso de prensado (Rivela et al., 2006; Wilson, 2010).

Por lo tanto, con el objetivo de que el material a desarrollar posea bajo impacto ambiental, se propone analizar el ciclo de vida del proceso de diseño y conformado del material para identificar en qué etapa se encuentra el mayor impacto ambiental. Se tomó como referencia la norma ISO 14040, titulada en español "Gestión ambiental. Análisis de ciclo de vida. Principios y marco de referencia (ISO 14040:2006)", la cual describe los principios y el marco de referencia para el análisis del ciclo de vida (AENOR, 2006). Se realizó el diagrama de ciclo de vida el cual se presenta en Figura 2 y se definió como unidad funcional un módulo del material de $150 \times 70 \times 5 \mathrm{~mm}$, compuesto por cáscaras de cuatro nueces. Se consideran como etapas a evaluar la materia prima, producción y transportes asociados.

\section{Discusión y resultados}

Los resultados iniciales (Figura 3) muestran que la cáscara de nuez se puede aglomerar de forma eficiente, donde mayoritariamente en cuanto a porcentaje 

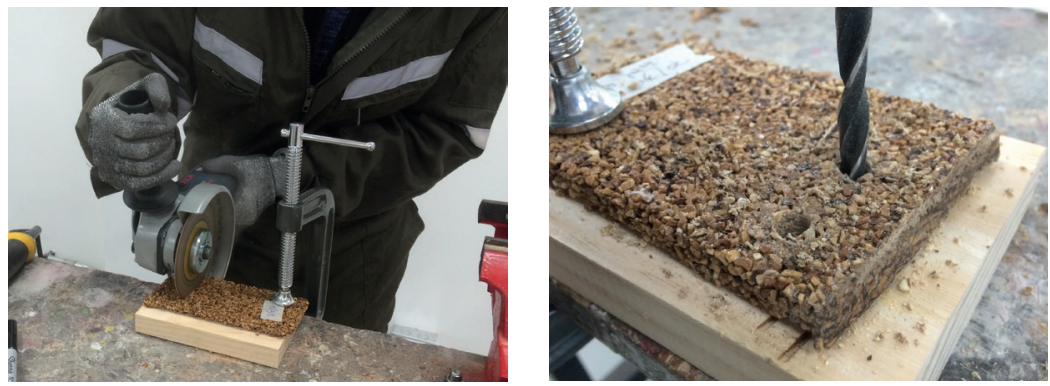

Figura 4. Izquierda: Corte con esmeril. Derecha: Perforación taladro manual. Fuente: Parodi Miranda, 2016.

el compuesto del material es el residuo agroindustrial. Por lo tanto, se obtiene como resultado la estandarización del procesamiento de un material compuesto en base a una granulometría de cáscara y un aglutinante biodegradable.

\section{Ensayos}

El compuesto posee una densidad aproximada de $950 \mathrm{~kg} / \mathrm{m}^{3}$. En cuanto al hinchamiento y absorción de agua, tal como se esperaba dado que el aglomerante es hidrosoluble, las probetas no pueden ser manipuladas ni evaluadas pues se deshacen en el agua.

En lo referido al rendimiento mecánico, se observa que, al momento de la fractura, la propagación corre de manera discontinua rompiéndose de a poco a lo largo del tiempo, esto ocurre por los granos que posee la muestra. El módulo elástico presenta un valor muy bajo, lo que significa que el material tiene un comportamiento elástico. Las bajas propiedades mecánicas de la muestra podrían deberse a la discontinuidad material del compuesto.

El compuesto estudiado, como se demostró a partir de las pruebas de mecanizado (Figura 4), puede ser conformado a partir de arranque de viruta, sin embargo, es un proceso que deja desperdicios. El esmeril permite realizar cortes en las muestras, sin desprender trozos. En el caso de la sierra ingleteadora, el corte se logra, sin embargo, se desprenden trozos, por lo cual se requiere un paso de trabajo posterior para un mejor acabado. La sierra de huincha, por su parte, deja un corte parejo y bien acabado. Finalmente, el corte láser deja la zona de corte con un color más oscuro al quemarse, lo cual es normal en los distintos materiales, pero funciona perfectamente. Con el taladro de manual y de pedestal, las perforaciones de 3 y $7 \mathrm{~mm}$ no quedan circulares pues se desprenden granos, de todas maneras, es factible realizarlos. Por otra parte, la conductividad térmica es una propiedad física de los materiales, tiene que ver con la capacidad de transferir calor de una partícula a otra por medio del contacto, se relaciona con la densidad, la temperatura y la humedad del material. Es importante rescatar que aquellos materiales que presenten un bajo valor de conductividad térmica serán malos conductores, lo que quiere decir aislantes, lo cual es el caso del material desarrollado y el cual posee un valor de conductividad de $0,068 \mathrm{~W} / \mathrm{mk}$, lo que lo posiciona como mejor aislante térmico que el yeso cartón (0,24 W/mk), adobe (o,9 W/mk), algunas maderas y bastante similar al aserrín de madera (0,06 W/mk) (INN, 1991).

\section{Manufactura}

Al buscar un material que cierre su ciclo de vida de manera circular, de tal manera que no genere residuos en sus procesos, se explora un proceso sin arranque de viruta, lo que significa trabajar la mezcla a partir de moldeado, lo cual da excelentes resultados. Se demuestra que se pueden desarrollar desde 
Figura 5. Izquierda: Resultado de molde media esfera. Derecha: Resultado de doble curvatura. Figura 6. Resultado análisis perceptual. Fuente: Parodi Miranda, 2016.
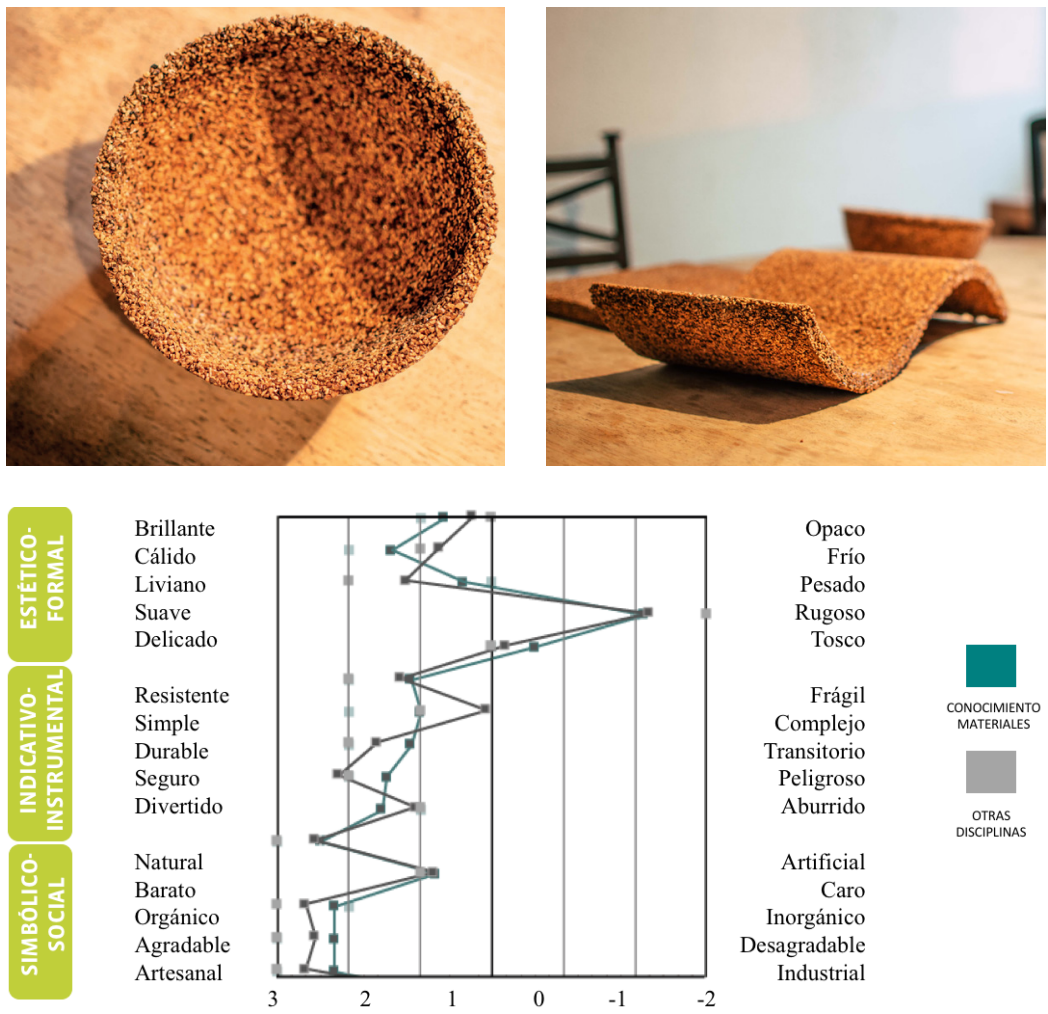

morfologías simples hasta dobles como se presenta en la Figura 5 y también triples curvaturas. Se ha identificado que mientras mayor es el espesor se consigue una mejor conformación, acabado y resistencia. El uso de un molde machihembrado y prensado permite una conformación compacta y bien definida, lo que entrega la posibilidad de innovar en la forma que adoptará. Respecto del análisis perceptual, se analiza el total de la muestra y como se observa en la Figura 6, existe en líneas generales una tendencia marcada para el lado izquierdo donde se ubican los atributos positivos, y gran tendencia hacia el adjetivo rugoso.

En general, a las personas les agrada el material, tienen la inquietud de saber de qué está conformado, lo huelen, lo manipulan. Además, en cuanto a la muestra, a pesar de que la mitad poseía conocimientos previos sobre materiales y los demás no, no se presentan diferencias significativas entre ambas. En cuanto al cierre de ciclo, luego de observar las probetas que se mantuvieron en un espacio interior cerrado como el hogar, versus las que se introdujeron en vermicompostaje y en macetero al aire libre, se ha verificado que el material puede permanecer varios meses en un espacio interior sin sufrir modificaciones, pero al estar en el exterior hay factores que facilitan su degradación. En ambos casos de exposición al exterior, las probetas, ya sea en macetero o con lombrices, comienzan un proceso de degradación, por lo tanto, se puede deducir que es un material que en su fin de vida no genera residuos y es respetuoso con el medio ambiente.

Finalmente, se ha llevado a cabo un primer acercamiento al análisis de ciclo de vida, considerando la etapa de diseño del material que entrega datos cuantitativos, que permiten observar impactos ambientales. Los resultados indican que la mayor ineficiencia se encuentra en la etapa de producción, 
esto dado por la aplicación de calor que se utiliza para secar la mezcla, lo cual tiene bastante relación con lo que ya se había estudiado en referentes. Sin embargo, el valor de detectar esta información en esta etapa temprana es que permite tomar decisiones en el diseño del material. No obstante, se trata de un proceso iterativo y que debe ser completado y evaluado nuevamente una vez que se hayan definido las fases que hoy se encuentran fuera de la frontera, en ese momento podrá ser comparado con otros materiales que cumplan la misma función.

\section{Conclusiones}

La presente investigación cumplió el objetivo de desarrollar un material compuesto y mediante la realización de ensayos se le logró caracterizar, al obtener las bases para proponer aplicaciones de diseño.

Luego de realizar la investigación, en cuanto a los resultados, se puede decir que visualmente presenta cohesión interna, la sensación al tacto es una textura rugosa; en cuanto al olor, este es suave y característico de la madera, el color de la cáscara no varía pues el aglomerante es translúcido. Además, el proceso de conformación a partir de moldes coincide con los requerimientos del material; respecto a costos, estos son bajos siendo más rentable a la hora de utilizarlo para grandes volúmenes; en lo que se refiere a impactos ambientales, produce poco o nada de residuos y si los moldes son fabricados en materiales duraderos, su impacto ambiental no es significativo. Por otra parte, los ensayos térmicos y pruebas de mecanizado muestran que es un material isotrópico, liviano y frágil, con destacadas características de aislación térmica, similar a una plancha de corcho y mejor aislante que diversas maderas, adobe, yeso cartón, entre otros.

El material es frágil. Mecánicamente, presenta características que lo asemejan principalmente a maderas de baja densidad y espumas. Sin embargo, a diferencia de los mencionados, el material compuesto en base a cáscara de nuez utiliza materiales locales, de bajo costo, no es tóxico y necesita bajo nivel de energía y agua para su procesamiento.

Respecto al fin de vida del material, cumple un ciclo circular cerrado, al finalizar su vida útil al ser hidrosoluble puede reintegrarse al medio natural.

Todas las propiedades mencionadas le otorgan ventajas claves para innovar en su aplicación, abriendo un amplio camino para reemplazar materiales existentes y desarrollar nuevos productos.

Dadas las características ya mencionadas, se pueden proponer aplicaciones del material en objetos de interior, contenedores, como recubrimiento o para uso en mobiliario, siempre y cuando sea acompañado de otros materiales que aporten estructura.

Luego de descubrir que es posible cortar el material con corte láser CNC, se abre un nuevo camino para investigar y experimentar posteriormente su manufactura con otras de las tecnologías digitales actuales. Para futuras investigaciones, se sugiere realizar ensayo de biodegradabilidad bajo norma, por otra parte, también se debe trabajar en la manera de producir industrialmente el material, considerándose cambiar el tipo de energía utilizada y/o la forma de secar la mezcla, dado que posee el mayor impacto ambiental. 


\section{Agradecimientos}

Agradecimientos a los laboratorios de Ingeniería Mecánica de la Universidad Tecnológica Metropolitana (UTEM), al Laboratorio de Climatización de la Universidad de Santiago de Chile (USACH), por permitir utilizar sus instalaciones para realizar los ensayos del material y a Felipe Colomer por su apoyo fotográfico.

\section{Referencias}

AENOR. (2006). UNE-EN ISO 14040:2006. Gestión ambiental. Análisis de ciclo de vida. Principios y marco de referencia. (ISO 14040:2006).

Aros, M., Narváez, G., \& Aros, N. (2009). "El diferencial semántico para la disciplina del diseño una herramienta para la evaluación de productos". Paper presented at the XIII Congreso Internacional de Ingenierìa de Proyectos.

ASTM. (1999). Standard Test Method for Evaluating Properties of Wood-Base Fiber and Particle Panel Materials. In (Vol. D 1037-99). West Conshohocken, PA, USA: American Society for Testing and Materials International.

ASTM. (2011). Standard Test Methods for Conducting Machining Tests of Wood-Base Panel Materials Methods of testing wood-base panels In (Vol. D1666-11). West Conshonhocken, USA: American Society for Testing and Materials International. .

Brundtland, G. H. (1987). Brundtland report. Our common future. Oxford University.

Calkins, M. (2009). Materials for sustainable sites a complete guide to the evaluation, selection, and use of sustainable construction materials. Hoboken, N.J.: Hoboken, N.J: Wiley.

Capuz, S., \& Gómez, T. (2004). Ecodiseño, ingeniería del ciclo de vida para el desarrollo de productos sostenibles. Valencia: Universidad Politécnica de Valencia.

Formaldehyde Emissions Standards for Composite Wood Products, 2070-AJ44 C.F.R. (2016).

FAO. (2017). Production quantities of Peaches and nectarines by country 2014. Crops. Recuperado de : http://www.fao.org/faostat/en/ - data/QC/visualize

Fundación para la Innovación Agraria, m. d. a. (2007). Resultados y Lecciones en Manejo de Nogales. Santiago: Gobierno de Chile.

Guzmán, P. (2016) Entrevista Rinconada Walnut/Interviewer: D. Parodi Miranda.

IdeaConsultora. (2012). Actualización del catastro de la agroindustria hortofruticola chilena. Recuerpado de: Santiago: http://www.odepa.cl/wp-content/files_mf/ 1383165686Catastro_Agroindustrial_Informe_final.pdf Instituto Nacional de la Normalización. (1991). NCh 853. Of91, "Acondicionamiento térmico-Envolvente térmica de edificios-Cálculo de resistencias y transmitancias térmicas". International, F. o. t. e. a. f. a. f. (2002). Good wood guide. Forestry \& British Timber. In. iQonsulting. (2016). "Agregación de valor de frutos secos, nueces y almendras". In.

Karana, E., Pedgley, O., \& Rognoli, V. (2014). Materials experience; fundamentals of materials and design. Oxford: Butterworth Heinemann.

Lemus, G. (2001). El nogal en Chile (G. Lemus Ed.) Santiago: Gobierno de Chile

Magin, G. (2002). The Good wood guide, London and Cambridge, Friends of the Earth; Fauna and Flora International. In. London, Cambridge.

Marlenin, R. (2011). Utilización de la cáscara de nuez chandler en el yeso. (Máster en Innovación Tecnológica en Edificación Máster), Madrid: Universidad Politécnica de Madrid, Madrid. Retrieved from http://oa.upm.es/10065/2/TESIS_MASTER_MARLENIN_MARTE_ROSARIO.pdf (10065)

McDonough, W., \& Braungart, M. (2005). Cradle to Cradle, rediseñando la forma en que hacemos las cosas. Nueva York: McGraw Hill

Ndazi, B., Tesha, J., \& Bisanda, E. (2006). Some opportunities and challenges of producing bio-composites from non-wood residue. Journal of materials science, 41.

Nitin, S., \& Singh, V. (2013). Mechanical behavior of walnut reinforced composite. J. Mater. Environ. Sci, 4(2), 238.

Parodi Miranda, D. (2016). Desarrollo de un material compuesto biodegradable a partir de la utilización del residuo cáscara de nuez. (diseñadora industrial), Santiago: Universidad de Chile.

Pirayesh, H., Khazaeian, A., \& Tabarsa, T. (2012). The potential for using walnut (Juglans regia $L$.) shell as a raw material for wood-based particleboard manufacturing. Composites Part B: Engineering, 43(8), 3276-3280. doi: 10.1016/j.compositesb.2012.02.016

Ramirez, M., \& Wechsler, A. (2010). Materials development: an alternative career pathway for design graduates. Second International Conference of Design Education. Rivela, B., Hospido, A., Moreira, T., \& Feijoo, G. (2006). Life Cycle Inventory of Particleboard: A Case Study in the Wood Sector. The International Journal of Life Cycle Assessment, 11(2), 106-113. doi:10.1065/lca2005.05.206 
Rodríguez, R., Ramírez, A., Palacios, H., Fuentes, F., Silva, J., \& Saucedo, A. (2015). Características anatómicas, físico-mecánicas y de maquinado de la madera de mezquite (Prosopis velutina Wooton). Revista Mexicana de Ciencias Forestales, 6.

Romero, O., López, R., Damián, M., Hernández, I., Parraguirre, J., \& Huerta, M. (2012). Evaluación del residuo de cáscara de nuez (Juglans regia L.) en la producción de plántulas de Pinus patula, en vivero. Agronomía Costarricense, 103-110.

Sellao, D., \& Castillo, C. (2015). Diseño y montaje de un dispositivo experimental para el estudio de materiales constructivos ante una carga térmica convectiva.

Torres, P. (2010). Estudio de pre factibilidad técnico-económica de una planta de molienda de cáscara de nuez. Universidad de Santiago de Chile, Santiago.

Trujillo, A., \& Arias, L. (2013). El coco, recurso renovable para el diseño de materiales verdes. Entre Ciencia e Ingeniería, 14, 93-100.

Valencia, A. (2008). Materiales y procesos de manufactura en innovación. metodología para innovar desde nuevas perspectivas. Actas de diseño, 5, 243-246.

Wartelle, L., \& Marshall, W. (2000). Citric acid modified agricultural by-products as copper ion adsorbents. Advances in Environmental Research 4(1), 1-7. doi: 10.1016/S1093-0191(00)00002-2

Wilson, J. (2010). Life-Cycle Inventory of particleboard in terms of Resources, Emissions, Energy and Carbon. Wood and fiber science, 42, 90-106.

Çöpür, Y., Güler, C., Akgül, M., \& Taşçıoğlu, C. (2007). Some chemical properties of hazelnut husk and its suitability for particleboard production. Building and Environment, 42(7), 2568-2572. 